\title{
Robustness of Resilience Indicators for Water Distribution System
}

\author{
T.R. Neelakantan, A. Ariffa Parakath
}

\begin{abstract}
Water distribution through pipe networks is considered a significant in human civilization. The percentage of population served by these networks is increasing continuously and the reliability studies of these networks are given more importance. In the recent years, resilience a form of reliability of the system, of water distribution networks, gained significant focus. Some of the popular resilience indicators developed by researchers are critically analyzed in this work. The limitations of the resilience indicators presented in this paper indicate the necessity to improve the indicators and develop new robust indicators.
\end{abstract}

Keywords : performance indicators, resilience, water distribution network.

\section{INTRODUCTION}

M unicipal water supply by pipe networks is popular for the past century. Research on improving the performance of water supply through the pipe network has significantly advanced in this period. One of the recent researches in the water supply field is using resilience indicators in the design and operation of water distribution networks. Though performance indicators like reliability, connectivity, etc., were used for nearly 50 years, the usage of resilience was seen only from the year 2000. Resiliency can be described in so many ways. Two major kinds of resiliency can be (1) based on the restoration time once the system falls into failure mode; and (2) based on the potential of the system to absorb a shock and function without much difference in performance. The second kind is investigated in the majority of the researches in this field. The objective of this paper is to critically analyze three of the resilience indicators so that the outcomes will be useful for future research.

\section{RESILIENCE INDEX}

One of the major turning points in water distribution network investigations, particularly with resilience focus, was due to the introduction of an indicator called Resilience Index by Todini [1] as follows.

Revised Manuscript Received on December 05, 2019.

* Correspondence Author

T.R. Neelakantan*, Department of Civil Engineering, Kalasalingam Academy of Research and Education, Krishnankoil - 626126, India, neelakantan@klu.ac.in

A. Ariffa Parakath, Department of Civil Engineering, Kalasalingam Academy of Research and Education, Krishnankoil - 626126, India, ariffaparakath24@gmail.com

$$
R I=\frac{\sum_{j=1}^{N} q_{j}^{*}\left(h_{j}-h_{j}^{*}\right)}{\sum_{i=1}^{R} Q_{i} H_{i}-\sum_{j=1}^{N} q_{j}^{*} h_{j}^{*}}=\frac{\text { excess power available }}{\text { excess power supplied }}(1)
$$

In the above equation, $N$ is number of demand nodes, $q_{j}^{*}$ is the demand and $h_{j}$ is the actual head at node $j, h_{j}^{*}$ is the minimum required head for supplying the demand, $R$ is the number of reservoirs or over-head tanks, $Q_{i}$ is the flowrate from reservoir $i$ and $H_{i}$ is the head which the water is from the reservoir node. The attempts to verify the results of Todini (2000) in this study revealed that the numerical values reported in [1] are not perfect. For example, [1] reported in table 3 for the two-loop network (Fig. 1) that the resilience index of Optimum Cost case and Set A as 0.22 and 0.41 respectively. However, these values work to 0.210 and 0.396 respectively. This is reported here as the new researchers can avoid wasting time in the verification. The details of the calculations are presented in tables 1 and 2 .

\section{NETWORK RESILIENCE INDEX}

Prasad and Park [2] proposed Network Resilience Index by introducing an additional factor in the original resilience index as follows.

$$
N R I=\frac{\sum_{j=1}^{N} C_{j} q_{j}^{*}\left(h_{j}-h_{j}^{*}\right)}{\sum_{i=1}^{R} Q_{i} H_{i}-\sum_{j=1}^{N} q_{j}^{*} h_{j}^{*}}
$$

in which

$$
C_{j}=\frac{\sum_{i=1}^{n p j} D_{i}}{\left(n p j \times \max \left\{D_{i}\right\}\right)}
$$

and $n p j$ is the number of pipes connected to a node. The logic of using $C_{j}$ is justified by stating that reliable loops can be ensured by the uniformity of diameters of the pipes connected to a node. $C_{j}$ is referred as uniformity coefficient.

The Network Resilience Index [2] lacks physical meaning compared to Todini's Resilience Index as $C_{j}$ is an arbitrary weight. In this context, Atkinson [3] suggested that apart from diameter, 
Table- I: Recalculation of Todini (2004) results presented in table 3 for optimal cost of $0.419 \times 10^{6}$

\begin{tabular}{|c|c|c|c|c|c|c|c|c|c|}
\hline Pipe No. & $\begin{array}{c}\text { Diameter } \\
\text { (in) }\end{array}$ & $\begin{array}{c}\text { Diameter } \\
(\mathbf{m m})\end{array}$ & Node No. & $\begin{array}{c}\mathbf{q}^{*} \\
\mathbf{m}^{\mathbf{3}} \mathbf{h}\end{array}$ & $\begin{array}{c}\boldsymbol{h}^{*} \\
\mathbf{m}\end{array}$ & $\begin{array}{c}\boldsymbol{h} \\
\mathbf{m}\end{array}$ & $\begin{array}{c}\boldsymbol{p}=\left(\boldsymbol{h}-\boldsymbol{h}^{*}\right) \\
\mathbf{m}\end{array}$ & $\begin{array}{c}\boldsymbol{q}^{*} \boldsymbol{p} \\
\mathbf{m}^{\mathbf{4}} / \mathbf{h}\end{array}$ & $\begin{array}{c}\boldsymbol{q}^{*} \boldsymbol{h}^{*} \\
\mathbf{m}^{\mathbf{4}} \mathbf{h}\end{array}$ \\
\hline 1 & 18 & 457.2 & 1 & 1120 & - & 210.00 & 210.00 & 235200.0 & 235200 \\
\hline 2 & 10 & 254.0 & 2 & 100 & 180 & 203.25 & 23.25 & 2325.0 & 18000 \\
\hline 3 & 16 & 406.4 & 3 & 100 & 190 & 190.46 & 0.46 & 46.0 & 19000 \\
\hline 4 & 4 & 101.6 & 4 & 120 & 185 & 198.45 & 13.45 & 1614.0 & 22200 \\
\hline 5 & 16 & 406.4 & 5 & 270 & 180 & 183.81 & 3.81 & 1028.7 & 48600 \\
\hline 6 & 10 & 254.0 & 6 & 330 & 195 & 195.44 & 0.44 & 145.2 & 64350 \\
\hline 7 & 10 & 254.0 & 7 & 200 & 190 & 190.55 & 0.55 & 110.0 & 38000 \\
\hline 8 & 1 & 25.4 & & & & & & & \\
\hline
\end{tabular}

Table- II: Recalculation of Todini (2004) results presented in table 3 for solution A with a cost of $0.450 \times 10^{6}$

\begin{tabular}{|c|c|c|c|c|c|c|c|c|c|}
\hline Pipe No. & $\begin{array}{c}\text { Diameter } \\
\text { (in) }\end{array}$ & $\begin{array}{c}\text { Diameter } \\
(\mathbf{m m})\end{array}$ & Node No. & $\begin{array}{c}\boldsymbol{q}^{*} \\
\mathbf{m}^{\mathbf{3}} / \mathbf{h}\end{array}$ & $\begin{array}{c}\boldsymbol{h}^{*} \\
\mathbf{m}\end{array}$ & $\begin{array}{c}\boldsymbol{h} \\
\mathbf{m}\end{array}$ & $\begin{array}{c}\boldsymbol{p}=\left(\boldsymbol{h}-\boldsymbol{h}^{*}\right) \\
\mathbf{m}\end{array}$ & $\begin{array}{c}\boldsymbol{q}^{*} \boldsymbol{p} \\
\mathbf{m}^{\mathbf{4}} \mathbf{h}\end{array}$ & $\begin{array}{c}\boldsymbol{q}^{*} \boldsymbol{h}^{*} \\
\mathbf{m}^{\mathbf{4} / \mathbf{h}}\end{array}$ \\
\hline 1 & 18 & 457.2 & 1 & 1120 & & 210.00 & 210.00 & 235200.0 & 235200 \\
\hline 2 & 16 & 406.4 & 2 & 100 & 180 & 203.25 & 23.25 & 2325.0 & 18000 \\
\hline 3 & 14 & 355.6 & 3 & 100 & 190 & 200.19 & 10.19 & 1019.0 & 19000 \\
\hline 4 & 6 & 152.4 & 4 & 120 & 185 & 198.38 & 13.38 & 1605.6 & 22200 \\
\hline 5 & 14 & 355.6 & 5 & 270 & 180 & 196.19 & 16.19 & 4371.3 & 48600 \\
\hline 6 & 1 & 25.4 & 6 & 330 & 195 & 195.99 & 0.99 & 326.7 & 64350 \\
\hline 7 & 14 & 355.6 & 7 & 200 & 190 & 191.35 & 1.35 & 270.0 & 38000 \\
\hline 8 & 10 & 254.0 & & & & & & & \\
\hline
\end{tabular}

pipe length, roughness, and upstream and downstream pressure heads can also be used for the network operation. Reference [4] also specified this in their review paper. Todini and his team criticized that NRI corrupts the original physical meaning of the resilience index [5]. Extending [3], more logical uniformity coefficients can also be defined in terms of area of cross section or length weighted diameter as described below.

$$
C_{j}=\frac{\sum_{i=1}^{n p j} A_{i}}{\left(n p j \times \max \left\{A_{i}\right\}\right)} \text { or } C_{j}=\frac{\sum_{i=1}^{n p j} L_{i} D_{i}}{\left(n p j \times \max \left\{L_{i} D_{i}\right\}\right)}
$$

The proposers of the Network Resilience Index neither explored nor explained why they have not considered the other forms as above. If a network has a (main source) pipe from storage splitting into three branches at a node, the pipes need not be of uniform diameter. Since there is only one inflow pipe and there are three outflow pipes at the junction node, the main source pipe can be of higher diameter; while the branching pipes may be of smaller size. In the 2-loop network at node two, there are three pipes (1,2 and 3) connected to it (Fig. 1). Among these three, pipe1 is from the reservoir and the other two are the branching pipes. During the non-failure scenario, pipes 2 and 3 share the flow from pipe1. However, if pipe 2 fails, the supply can follow through pipe3 and vice-versa. Hence, generally maintaining uniformity of pipe diameters is dispensable or not required.

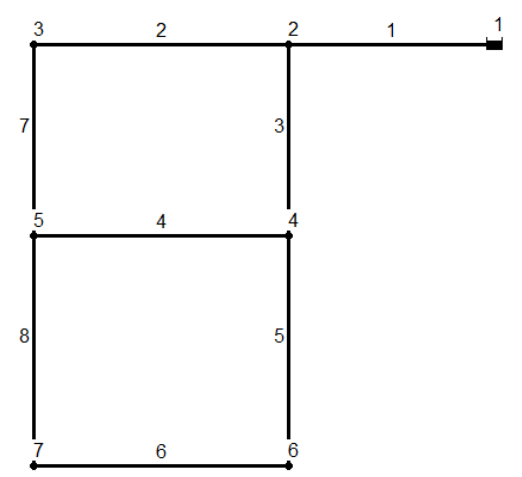

Fig. 1. Two-loop network.

\section{TOTAL SURPLUS EAD INDEX}

Reference [2] proposed a resilience index called 'Total Surplus Head Index' $\left(I_{t}\right)$ which is the summation of the surplus heads at demand nodes and is presented below in the mathematical form.

$$
I_{t}=\sum_{i=1}^{N}\left(H_{i}-H_{i}^{l}\right) \quad \text { for all } i=1,2, \ldots N
$$

In the above equation, $N$ is number of demand nodes, $H_{i}$ is the actual head at node $i$ and $H_{i}^{l}$ is the minimum required head for supplying the demand. They claimed that maximization of this index improves the ability of the distribution network. However, it has an issue which is explained here. The surplus head indicates available power or energy for dissipation during a failure event. Water distribution networks usually have different zones like low-pressure zone and high-pressure zone. If we have a larger number of nodes in a high-pressure region where the surplus heads are more, then the algebraic sum of all the surplus heads will provide a large total (large $I_{t}$ ). However, if we have a 
larger number of nodes in a low-pressure region, the algebraic sum of all the surplus heads will provide a small total (small $\left.I_{t}\right)$. During a pipe failure or large demand change, if a high-pressure region is going to get affected, the value of $I_{t}$ will fall drastically. Thus, the value of $I_{t}$ depends on the density of demand nodes in different regions and it will not reflect the resilience properly when the nodes are distributed unevenly.

Suppose if a demand node is made into two nodes each with $50 \%$ of original demand (total demand is same) and the distance between the nodes is very small, the head at these two nodes will be equal to the head obtained with one node with $100 \%$ demand. Thus, when a node is split into two nodes the Total Surplus Head Index will also increase. When nodes are increased, the Total Surplus Head Index will also increase. This index is good only to compare a network with different diameter sets. However, a network cannot be compared with another network based on this index. The value for this depends on the density of demand nodes in the network. Nodes placed densely provide higher value for Total Surplus Head Index. To avoid this, weights may be attached to the nodes. For example, if a node is split into two as explained above, each divided node cannot have a weight equal to the original combined node. The natural weight for nodes can be the supply (or demand) from the node. When a node is split into two nodes as explained above, since the demands are also divided into $50 \%$, a weight of 0.5 to each can be considered. For the whole network, this can be expanded such that each demand node can be given a weight value equal to demand or demand ratio to the total demand. When demand is given as weight, it leads to the power concept. Weighted summation provides a function of total power coming out of the network or the weighted summation multiplied with weight density of water provides the total power coming out of the network. The ratio of total power coming out of the network and total power imparted to the network (flow from reservoir or over-head-tank multiplied by the head multiplied by weight density of water + pump power if any) provides dimensionless number indicating resilience. A higher ratio indicates higher resilience. Though not explained as above, relating to Total Surplus Head Index of [2], this ratio was used earlier as power efficiency [6, 7] and as Available Power Index [8]. Though [8] claim that the Available Power Index is a new index developed, the same index is reported in literature earlier [6, 7].

\section{CONCLUSION}

In this work, three important resilience indicators, popularly used in the water distribution network analysis in the last two decades, are critically analyzed analytically, and the issues identified are discussed. From the analyses, it has been identified than resilience index proposed in [1] is better than the other two popular indicators developed later. The results further indicate the need for a more robust resilience indicator for water distribution networks.

\section{REFERENCES}

1. E. Todini, "Looped water distribution networks design using a resilience index based heuristic approach," Urban Water, 2000, vol. 2(2), pp. $115-122$.

2. T.D. Prasad, and N.S. Park, "Multiobjective genetic algorithms for design of water distribution networks," J. Water Resour. Plann. Manage., 2004, 10.1061/(ASCE)0733-9496

3. S. Atkinson, "A futures approach to water distribution and sewer network (re) design," Ph.D. dissertation, Univ. of Exeter, U.K., 2013.

4. A. Gheisi, M. Forsyth, G. Naser, "Water distribution systems reliability: A review of research literature," J. Water Resour. Plan. Manag., 2016, vol. 142, 04016047

5. E. Creaco, M. Franchini, and E. Todini, "Generalized Resilience and Failure Indices for Use with Pressure-Driven Modeling and Leakage," J. Water Resour. Plann. Manage., 2014, vol. 142(8), 10.1061/(ASCE)WR.1943-5452.0000656.

6. C.R. Suribabu, T.R. Neelakantan, "Sizing of water distribution pipes based on performance measure and break-repair-replacement economics," ISH J Hydraul Eng., 2012, vol. 18(3), pp. 241-251.

7. C.R. Suribabu, K. Prashanth, S. Vignesh Kumar, N. Sai Ganesh, "Resilience enhancement methods for water distribution networks," Jordon J Civ Eng., 2016, vol. 10(2), pp. 216-231.

8. H. Liu, D.A. Savic, Z. Kapelan, E. Creaco, Y. Yuan, "Reliability Surrogate Measures for Water Distribution System Design: Comparative Analysis," J. Water Resour. Plann. Manage., 2017, vol. 143(2), 10.1061/(ASCE)WR.1943-5452.0000728.

\section{AUTHORS PROFILE}

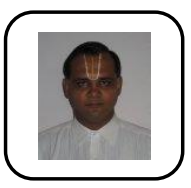

Dr. T.R. Neelakantan is a Senior Professor in Civil Engineering and Director - Accreditation and Ranking a Kalasalingam Academy of Research and Education, Tamilnadu. He obtained his Ph.D. degree in Civil Engineering from Anna University in the year 1998. His working experiences are from Anna University, IIT-Madras, SASTRA Deemed University and the University of Kentucky, Lexington, USA. He published more than 60 articles in reputed journals, and handled many government and private funded projects both in India and USA.

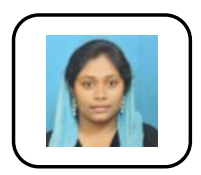

A. Ariffa Parakath was a PG students of Department of Civil Engineering, Kalasalingam Academy of Research and Education and completed her M.Tech. course in the academic year 2018-19. 